\title{
Epigenome-wide data collection in a case of gliofibroma
}

\author{
Felix Behling ${ }^{1,2,3}$, Florian Hennersdorf ${ }^{4}$, Jens Schittenhelm²,3,5 \\ ${ }^{1}$ Department of Neurosurgery, Eberhard Karls University, Tübingen, Germany, ${ }^{2}$ Comprehensive Cancer Center Tübingen, Eberhard \\ Karls University, Tübingen, Germany, ${ }^{3}$ German Cancer Consortium (DKTK), DKFZ partner site Tübingen, Germany, ${ }^{4}$ Department \\ of Diagnostic and Interventional Neuroradiology, Eberhard Karls University, Tübingen, Germany, ${ }^{5}$ Department of Neuropathology, \\ Eberhard Karls University, Tübingen, Germany
}

\begin{abstract}
Gliofibroma is a rare tumour entity with glial and mesenchymal histological features. We describe the case of a 30-year-old woman who presented with a short history of intermittent left-sided facial pain and paraesthesia of the left upper extremity. Histologically, the tumour consisted of a mixture of glial fibrillary acidic protein (GFAP)-positive glial cells and collagen-rich stroma. Immunohistochemical and molecular analysis showed no IDH1/2, BRAF, H3F3A mutations or ATP-dependent helicase (ATRX) loss in this tumour. Illumina Infinium HumanMethylation450 BeadChip array (HM450) methylation profile of the tumour was different from typical glioma entities. Genome-wide DNA copy number analysis showed partial loss of chromosome 3 and 8. All previous cases are reviewed. Our data support the classification of gliofibroma as a rare, but distinct brain tumour entity with good prognosis.
\end{abstract}

Key words: gliofibroma, epigenomic analysis, 450k methylation array, WHO classification, rare brain tumour.

\section{Introduction}

Gliofibroma is a rare tumour entity. Only 43 cases including this patient have been reported to date in the English literature (Table I) [1-4,6,7,9-12,14-20, 24,26-31,33-39]. The first case was described by Friede in 1978 [11]. Its biphasic histological appearance comprises of glial and mesenchymal features. The glial component can vary between a low- and high-grade level of differentiation resulting in varying prognostic outlooks, while the mesenchymal part persistently shows benign behaviour $[10,12,35]$. However, one case was reported that progressed despite lacking histopathological signs of higher grade of its glial component. The majority of cases are described as low-grade and mostly affect younger patients within the first two decades of life. The age ranges from 11 days to 58 years $[12,15,19]$ (see Table I). Tumours are known to form in the hemispheres, as well as the cerebellum, the ventricles, the spinal cord and the brainstem. They are believed to develop de novo. As an exception, one case emerged from hamartoma-like lesions $[4,11,15,30]$ and another after treatment of a pilocytic astrocytoma [1]. Some gliofibromas show calcifications that can be quite pronounced and even lead to the first radiologic impression of meningioma $[19,20]$. Besides surgical resection, no clear management guidelines exist. Complete surgical excision has been described as an important form of treatment [12]. Eleven out 
Table I. List of all reported gliofibromas in the literature with the available clinical data

\begin{tabular}{|c|c|c|c|c|c|c|c|}
\hline No. & Author & Age & Sex & Location & Surgery & Adjuvant treatment & Outcome \\
\hline 1 & Friede & $3.9 \mathrm{y}$ & $\mathrm{F}$ & Brainstem & Autopsy & $\mathrm{RT} / \mathrm{CT}$ & 3 m (dead) \\
\hline 2 & $\begin{array}{l}\text { Budka and Sunder- } \\
\text { Plassmann }\end{array}$ & $45 y$ & $\mathrm{~F}$ & Spinal cord & PR & None & 1 y (alive) \\
\hline 3 & Iglesias et al. & $11 \mathrm{~d}$ & M & Spinal cord & PR & None & 4 y (alive) \\
\hline 4 & Reinhardt and Nahser & $16 y$ & $\mathrm{~F}$ & Cerebrum & $C R$ & None & $6 \mathrm{~m}$ (alive) \\
\hline 5 & Vázquez et al. & $9 y$ & $\mathrm{~F}$ & Spinal cord & PR & RT & $18 \mathrm{~m}$ (dead) \\
\hline 6 & Vázquez et al. & $5.6 y$ & M & Spinal cord & PR & RT & 2.5 y (alive) \\
\hline 7 & Vázquez et al. & $11 \mathrm{~m}$ & $\mathrm{~F}$ & Cerebrum & $\mathrm{PR}$ & None & 2 y (alive) \\
\hline 8 & Snipes et al. & $2 \mathrm{~m}$ & $\mathrm{~F}$ & Thalamus/posterior fossa & $\mathrm{PR}$ & None & $16 \mathrm{~m}$ (dead) \\
\hline 9 & Schober et al. & $18 \mathrm{y}$ & $M$ & Cerebrum & $C R$ & ND & ND \\
\hline 10 & Iglesias-Rozas et al. & $1.2 \mathrm{y}$ & $\mathrm{F}$ & Cerebrum & $C R$ & None & $18 \mathrm{~m}$ (alive) \\
\hline 11 & Cerda-Nicolas and Kepes & $9 y$ & $M$ & Cerebrum & $C R$ & ND & $5.5 \mathrm{~m}$ (alive) \\
\hline 12 & Cerda-Nicolas and Kepes & $4 y$ & $\mathrm{~F}$ & IV ventricle & Biopsy & ND & ND \\
\hline 13 & Rushing et al. & $6 \mathrm{~m}$ & $\mathrm{~F}$ & IV ventricle & $C R$ & None & 2 y (alive) \\
\hline 14 & Windisch et al. & $5 \mathrm{~m}$ & M & Spinal cord & PR & None & 7 m (alive) \\
\hline 15 & Caldenmeyer et al. & $8 y$ & M & Cerebrum & Biopsy & $\mathrm{CT}$ & ND (alive) \\
\hline 16 & Caldenmeyer et al. & $6 \mathrm{~m}$ & $\mathrm{~F}$ & Cerebellum & $C R$ & None & ND (alive) \\
\hline 17 & Prayson & $3 \mathrm{~m}$ & $M$ & Cerebrum & PR & None & 3 y (alive) \\
\hline 18 & Sharma et al. & $20 y$ & $M$ & Cerebrum & $C R$ & None & $1 \mathrm{y}$ (alive) \\
\hline 19 & Sharma et al. & $24 y$ & $\mathrm{~F}$ & Spinal cord & PR & None & 2 y (alive) \\
\hline 20 & Sharma et al. & $54 y$ & $M$ & Cerebrum & $C R$ & RT & $6 \mathrm{~m}$ (dead) \\
\hline 21 & Mölenkamp et al. & ND & ND & ND & ND & ND & ND \\
\hline 22 & Matsumara & $12 y$ & $F$ & Spinal cord & $C R$ & ND & 2.8 y (alive) \\
\hline 23 & Erguvan-Önal et al. & $16 y$ & $M$ & Cerebrum & $C R$ & None & 14 m (alive) \\
\hline 23 & Kim et al. & $25 y$ & $M$ & Cerebrum & $C R$ & None & $2 \mathrm{~m}$ (alive) \\
\hline 25 & Suárez et al. & $4 \mathrm{~m}$ & $M$ & Suprasellar & Biopsy & $\mathrm{CT}$ & 3 y (alive) \\
\hline 26 & Deb et al. & $15 \mathrm{y}$ & ND & Brainstem & CR & None & ND \\
\hline 27 & Nomura et al. & ND & ND & ND & ND & ND & ND \\
\hline 28 & Goyal et al. & $8 y$ & $M$ & Cerebrum & ND & $\mathrm{RT} / \mathrm{CT}$ & $1 \mathrm{y}$ (alive) \\
\hline 29 & Goyal et al. & $15 \mathrm{y}$ & $\mathrm{F}$ & III ventricle & PR & $\mathrm{RT} / \mathrm{CT}$ & 2 y (alive) \\
\hline 30 & Goyal et al. & $40 y$ & $M$ & Cerebrum & $C R$ & RT & 3 y (alive) \\
\hline 31 & Sarkar et al. & $3 \mathrm{~m}$ & $\mathrm{~F}$ & II/III ventricle & Biopsy & None & 10 y (alive) \\
\hline 32 & Prayson et al. & $19 y$ & $\mathrm{~F}$ & Spinal cord & ND & ND & ND \\
\hline 33 & Gargano et al. & $10.7 y$ & $\mathrm{~F}$ & Cerebrum & $C R$ & None & 2 y (alive) \\
\hline 34 & Escalante Abril et al. & $50 y$ & $\mathrm{~F}$ & Cerebrum & Biopsy & None & $1 \mathrm{~m}$ (dead) \\
\hline 35 & Jones et al. & ND & ND & ND & ND & ND & ND \\
\hline 36 & Jones et al. & ND & ND & ND & ND & ND & ND \\
\hline 37 & Jones et al. & ND & ND & ND & ND & ND & ND \\
\hline 38 & Jones et al. & ND & ND & ND & ND & ND & ND \\
\hline 39 & Kang et al. & $58 y$ & $\mathrm{~F}$ & Cerebrum & PR & None & 4 y (alive) \\
\hline 40 & Ahmad et al. & $23 y$ & $\mathrm{~F}$ & Brainstem & PR & RT & 3 y (alive) \\
\hline 41 & Amoroso et al. & ND & ND & ND & ND & ND & ND \\
\hline 42 & Kaneva et al. & $12 \mathrm{~m}$ & $M$ & Brainstem & PR & $\mathrm{RT} / \mathrm{CT}$ & $21 \mathrm{~m}$ (dead) \\
\hline 43 & Behling et al. & $30 y$ & $\mathrm{~F}$ & Cerebrum & $C R$ & None & 4.9 y (alive) \\
\hline
\end{tabular}

$C R$ - complete resection, $P R$ - partial resection, $C T$ - chemotherapy, $R T$ - radiotherapy, $d$ - days, $m$ - months, $y$-years, ND - no data available 
of twelve patients that initially received complete resection of the tumour lesion (Table I) were reported as being alive, while 2 patients were presented in the literature without information about the further clinical course. However, it is important to note that the reported follow-up intervals vary widely. As adjuvant treatment several chemotherapeutic agents have been applied. After lesion biopsy Suárez et al. successfully applied a vincristine and carboplatin regimen [37]. Goyal et al. suggest temozolomide as an adjuvant treatment for high-grade and recurrent gliofibromas [14]. Recently, a case harbouring a $v$-Raf murine sarcoma viral oncogene homolog $B$ (BRAF(V600E)) mutation received vemurafenib, which was reported to have stabilized the residual tumour for some time [18]. However, there are no sufficient data on the efficacy of chemotherapy in this rare tumour entity. Sarkar et al. even suggest conservative treatment if the histology is benign (case with the longest follow-up, 10 years alive) [33].

Due to its rarity, the development as well as the clinical features of gliofibroma remain poorly understood. To date it is not listed as a separate tumour entity in the World Health Organization (WHO) classification [22]. However, in the era of molecular diagnostics and classification of central nervous system
(CNS) tumours, it is necessary to reconsider the classification of rare tumours such as gliofibroma beyond the known histopathological characteristics. We therefore present a case of gliofibroma together with the histopathological and additional molecular data.

\section{Clinical summary}

A 30-year-old woman came to our outpatient clinic and presented with a short history of episodic left-sided facial tension. She also complained of intermittent paraesthesia of her left arm along dermatome C8 usually occurring in the morning. An magnetic resonance imaging (MRI) of the cervical spine revealed a disc prolapse in the segment $\mathrm{C} 5 / 6$. But electrophysiological assessment was unremarkable. In the further course she developed a pressure sensation behind her right eye accompanied by blurred vision, which led to a cranial MRI scan. It showed a space occupying the lesion in the superior frontal gyrus of the right hemisphere with strong peripheral contrast enhancement and central sparing, measuring approximately $8 \mathrm{~mm}$ in diameter (Fig. 1). MR spectroscopy was uncertain regarding the entity of the lesion. On the day of her first visit, her neurological exam was unremarkable. Her prior medical history comprised of a urethral
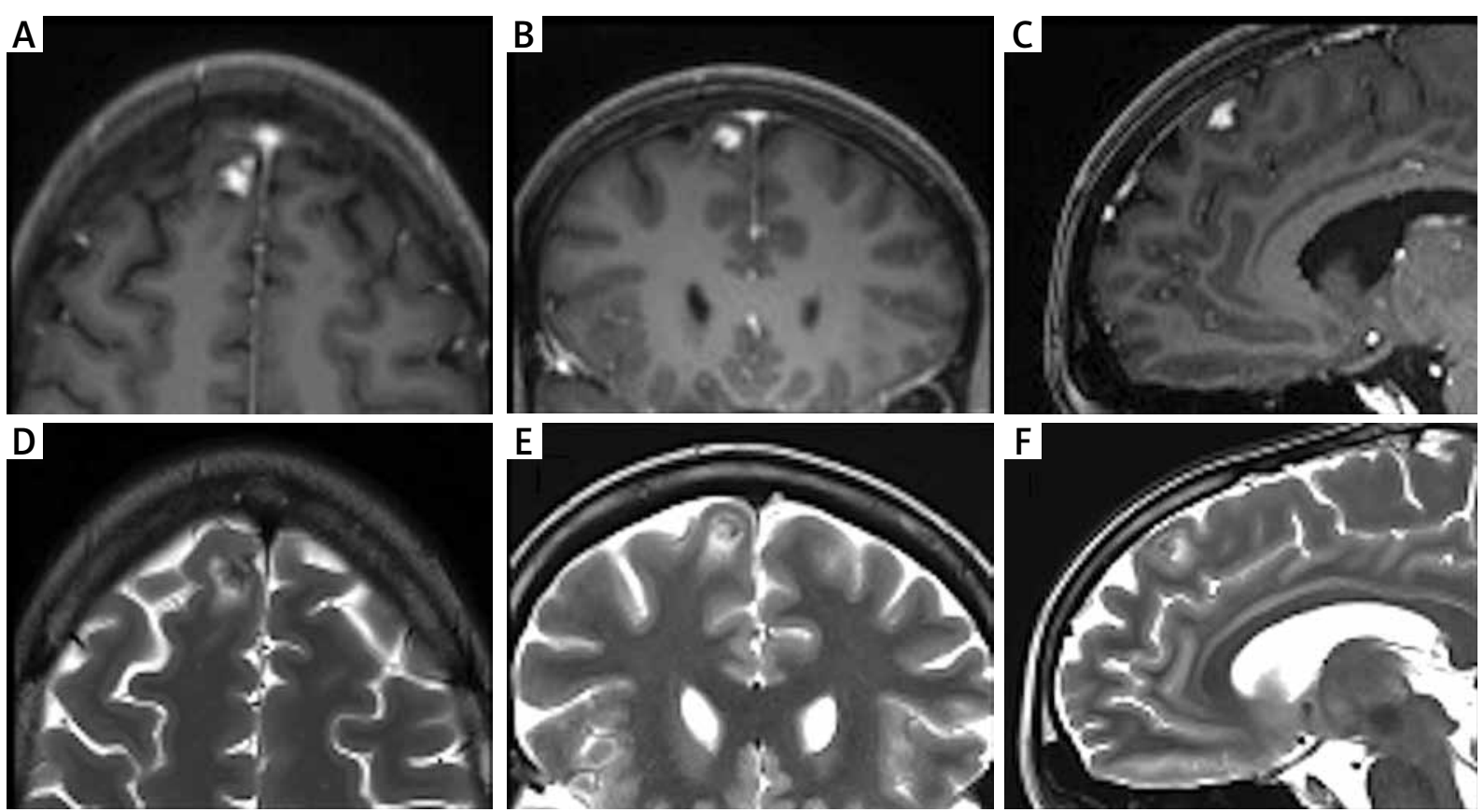

Fig. 1. Preoperative MRI. A-C) A small irregular intraparenchymal lesion with enhancement in postcontrast T1-weighted sequences (axial, coronal and sagittal sequences, respectively). D-F) Depict the corresponding T2-weighted images. 
stricture and mild hypothyroidism. Complete surgical resection was achieved. Intraoperatively, the tumour appeared as firm yellow-grey tissue. The operation as well as the postoperative course were uneventful, and the patient made a quick recovery. No adjuvant treatment was done. At the follow-up visit 4 years and 11 months after surgical resection she was in good clinical status and without neurological deficits. The MRI showed no signs of tumour recurrence.

\section{Pathology findings}

Histopathological evaluation of the tumour tissue showed a biphasic glial and mesenchymal pat- tern, fitting the diagnosis of gliofibroma. Reference assessments from the Department of Pathology in Düsseldorf and Bonn were attained and approved the diagnosis. Figure 2 illustrates the histopathological findings that led to the diagnosis of gliofibroma. A clear biphasic appearance was observed (Fig. 2A, B), with a glial (glial fibrillary acidic protein [GFAP]-positive, Fig. 2D) and a fibroblastic component (Elastica-van Gieson positive, Fig. 2H). MIB-1 immunopositivity was estimated to be below $3 \%$ with similar distribution among the different tumour compartments. Nuclear staining for P53 was seen in less than $1 \%$ of tumour cells. Cluster of differentia-
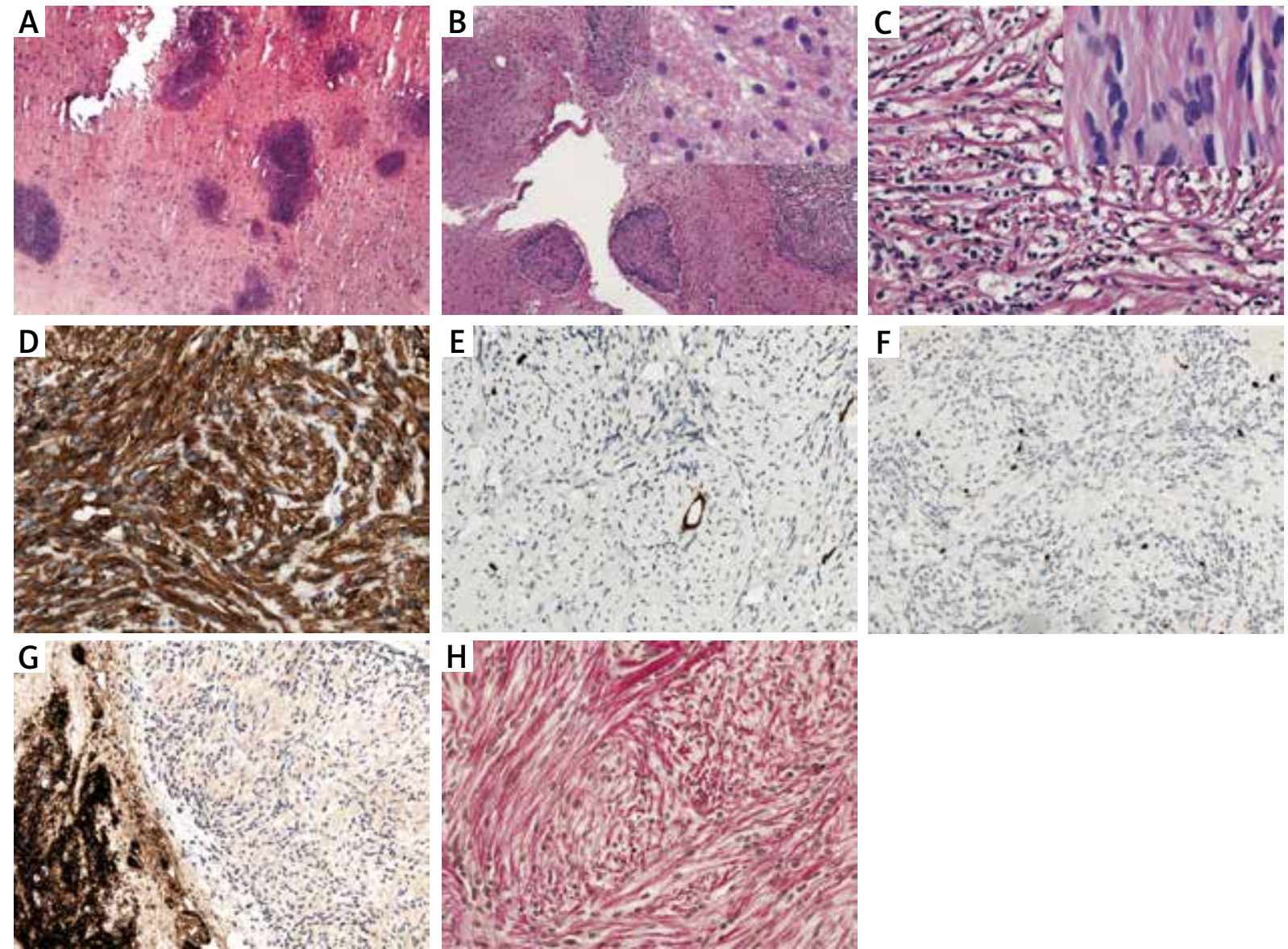

Fig. 2. Histopathological assessment. A) HE-staining of an intraoperative frozen section with multiple tumour lesions within reactive central nervous system (CNS) tissue. B) HE-staining after formalin fixation highlighting the infiltrative nature and biphasic pattern of the tumour. C) HE-staining with higher magnification shows rounded glial cells and alternating fibroblastic nuclei of mesenchymal deposits. D) GFAP immunostaining confirming the astrocytic nature of the glial component (brown colour). E) Except for endothelia, the tumour lacks immunoreactivity for CD34. F) MIB-1 staining with low proliferative activity (less than $3 \%$ of nuclei stained). G) Synaptophysin immunostaining with strong reaction of adjacent CNS tissue (left) but no clear staining of tumour cells (right). H) Elastica van Gieson staining highlights collagen within the tumour (red). Original magnification A, B, E, F: 100x, C, D: 200x, inlay for B, C: 400x. 
tion 34 (CD34) expression was restricted to endothelia. Synaptophysin and neurofilament were absent in the tumour. Epithelial membrane antigen (EMA) staining showed a paranuclear dot pattern in only few cells in isolated areas. Immunohistochemical staining showed no positive results for the isocitrate dehydrogenase (IDH)-1(R132H) and BRAF(V600E) mutation specific antibodies. Nuclear staining for oligodendrocyte transcription factor (Olig2) was absent while signal transducer and activator of transcription 6 (Stat6) was partially positive within tumour nuclei. No nuclear ATP-dependent helicase (ATRX) loss was seen in the tumour tissue. DNA isolation and subsequent sequencing of IDH1/2 exons 4 and H3F3A exon 1 showed wild-type sequences.

A 450k methylation array was done at the Department of Neuropathology of the University Hospital in Heidelberg. It showed partial loss of chromosome 3 and 8 in the copy number profile. There was no match with any of the established methylation class entities of the Neuropathology Brain Tumor classifier [5]. The closest similarity was seen to the methylation profile of schwannomas. The MGMT promoter was unmethylated.

\section{Discussion}

We describe a case of gliofibroma. Due to its rarity, very little information on clinical and molecular characteristics of this tumour entity exist. Immunohistochemistry and molecular analysis for IDH-1/2 and Olig2 were negative and there was nuclear staining for ATRX, indicating no loss. These findings argue for the astrocytic origin of gliofibroma, while Stat6 was partially positive indicating some astrocytic relation [25]. Several authors cited a source that supposedly discovered a loss of heterozygosis of chromosome 10 and 17 [12,24]. However, taking a closer look at the cited source, it clearly states different facts. First of all, the analysed tumour tissues were not gliofibromas but two desmoplastic infantile astrocytomas (DIA). Additionally, only chromosome 10 and 17 were checked for allelic loss and were found to be unharmed [23].

Of 42 cases described in the literature, to our knowledge this is the first time a 450k methylation array is reported. It showed a partial loss on chromosome 3 and 8 . The methylation array showed a slight similarity with the results found in schwannomas [5]. Interestingly, it has been suggested to rename this tumour type glioneurofibroma, based on observed Schwann cell like features of the mesenchymal com- ponent [38]. Another source described a histopathological variant of gliofibroma with signs of ependymoma, coining the term "desmoplastic ependymoma" [40]. It has also been proposed that gliofibroma and desmoplastic infantile ganglioglioma (DIG) should be regarded as the same tumour entity [31].

In fact, an important differential diagnosis that has to be considered when encountering an alleged gliofibroma is the desmoplastic infantile astrocytoma (DIA). Like gliofibroma it expresses fibroblastic and astrocytic features. In contrast, it has distinct clinical features. It typically shows dural attachment and forms large cysts. It mainly affects infants between the age of 1 to 24 months, and it shows a good clinical prognosis after surgical resection [22]. Gliofibroma, on the other hand, was discovered in patients ranging from infancy to older adulthood and may take a malignant course [10].

Even though complete surgical resection is widely viewed as the most important treatment, due to the small number of reported cases no clear management guidelines exist. A few cases received experimental chemotherapy regimens $[14,18,37]$. Interestingly, a loss on chromosome 8 has been reported in one case of DIA suggesting a genetic similarity to gliofibroma [21]. Recently, a detailed molecular characterization of 4 DIAs and 10 desmoplastic infantile gangliogliomas (DIGs) has been reported. Partial loss on chromosome 5, 10 and 21 was observed. Overall, there was no significant genetic pattern of difference between DIAs and DIGs. One case harbouring a BRAF mutation was identified [13]. Nevertheless, a very recent whole-genome sequencing-based study of different paediatric low-grade glioma revealed a new gene fusion involving fragile $X$-related protein 1 (FXR1) and BRAF in one investigated DIG case [41].

For further molecular insights into the tumour entity gliofibroma, it would be of great interest to attain separate molecular analyses of the glial and fibrous compartments to address the question whether the fibrous compartment is reactive or neoplastic in nature. However, since the compartmentalization is on a microscopic level, laser captured microdissection would be necessary in such cases.

The distinct clinical characteristics of gliofibroma and desmoplastic infantile astrocytoma underline the importance of distinguishing these two tumour entities. Even though some histopathological similarities exist, on the molecular level no clear similarities have been shown. According to literature reviews, especially 
immunohistochemical astrocytic markers are mostly absent in the mesenchymal component of gliofibroma [7]. Thus, the proposition to file gliofibroma and DIA/DIG as similar tumour types is not sustainable. We believe that gliofibroma should be viewed as an independent tumour entity and therefore be listed as such in the WHO classification. In light of the regular adjustments of the classification of low-grade gliomas on the basis of molecular insight [8,32], the classification of rare tumour entities such as gliofibromas needs to be considered for adjustments as well.

\section{Conclusions}

This rare case of gliofibroma is presented here to emphasize the differential diagnosis when encountering a tumour with fibroblastic and glial components. Further molecular investigations are necessary in order to form a more complete picture of the histopathological features of gliofibroma. The new molecular insights presented with this case underline that gliofibroma is not just a desmoplastic variant of a low-grade glioma but a distinct tumour entity that should be listed as such in the WHO classification.

\section{Acknowledgments}

We thank David Capper for providing the 450k methylation array.

\section{Availability of data and material}

Please contact the author for data requests.

\section{Consent for publication}

The informed consent to the scientific use of tissue and data was obtained from the patient.

\section{Disclosure}

The authors report no conflict of interest.

\section{References}

1. Ahmad MU, Barborie A, Pizer B, Husband D, Mallucci C, Jenkinson MD. Midbrain gliofibroma presenting in adulthood following "cure" of a childhood intraventricular pilocytic astrocytoma. Pediatr Neurosurg 2017; 52: 151-154.

2. Amoroso R, Weiss A, Pieri F, Zucchi V, Bosio M, Stasi I, Santonocito O. Fronto-mesial-pericallosal gliofibroma. J Neurosurg Sci 2020; 64: 302-304.

3. Budka H, Sunder-Plassmann M. Benign mixed glial-mesenchymal tumour ("glio-fibroma") of the spinal cord. Acta Neurochir (Wien) 1980; 55: 141-145.
4. Caldemeyer KS, Zimmerman RA, Azzarelli B, Smith RR, Moran CC. Gliofibroma: CT and MRI. Neuroradiology 1995; 37: 481-485.

5. Capper D, Jones DTW, Sill M, Hovestadt V, Schrimpf D, Sturm D, Koelsche C, Sahm F, Chavez L, Reuss DE, Kratz A, Wefers AK, Huang K, Pajtler KW, Schweizer L, Stichel D, Olar A, Engel NW, Lindenberg K, Harter PN, Braczynski AK, Plate KH, Dohmen H, Garvalov BK, Coras R, Holsken A, Hewer E, Bewerunge-Hudler M, Schick M, Fischer R, Beschorner R, Schittenhelm J, Staszewski O, Wani K, Varlet P, Pages M, Temming P, Lohmann D, Selt F, Witt H, Milde T, Witt O, Aronica E, Giangaspero F, Rushing E, Scheurlen W, Geisenberger C, Rodriguez FJ, Becker A, Preusser M, Haberler C, Bjerkvig R, Cryan J, Farrell M, Deckert M, Hench J, Frank S, Serrano J, Kannan K, Tsirigos A, Bruck W, Hofer S, Brehmer S, Seiz-Rosenhagen M, Hanggi D, Hans V, Rozsnoki S, Hansford JR, Kohlhof P, Kristensen BW, Lechner M, Lopes B, Mawrin C, Ketter R, Kulozik A, Khatib Z, Heppner F, Koch A, Jouvet A, Keohane C, Muhleisen H, Mueller W, Pohl U, Prinz M, Benner A, Zapatka M, Gottardo NG, Driever PH, Kramm CM, Muller HL, Rutkowski S, Von Hoff K, Fruhwald MC, Gnekow A, Fleischhack G, Tippelt S, Calaminus G,Monoranu CM, PerryA, JonesC,JacquesTS, Radlwimmer B, Gessi M, Pietsch T, Schramm J, Schackert G, Westphal M, Reifenberger G, Wesseling P, Weller M, Collins VP, Blümcke I, Bendszus M, Debus J, Huang A, Jabado N, Northcott PA, Paulus W, Gajjar A, Robinson GW, Taylor MD, Jaunmuktane Z, Ryzhova M, Platten M, Unterberg A, Wick W, Karajannis MA, Mittelbronn M, Acker T, Hartmann C, Aldape K, Schüller U, Buslei R, Lichter P, Kool M, Herold-Mende C, Ellison DW, Hasselblatt M, Snuderl M, Brandner S, Korshunov A, von Deimling A, Pfister SM. DNA methylation-based classification of central nervous system tumours. Nature 2018; 555: 469-474.

6. Cerda-Nicolas M, Kepes JJ. Gliofibromas (including malignant forms), and gliosarcomas: a comparative study and review of the literature. Acta Neuropathol 1993; 85: 349-361.

7. Deb P, Sarkar C, Garg A, Singh VP, Kale SS, Sharma MC. Intracranial gliofibroma mimicking a meningioma: a case report and review of literature. Clin Neurol Neurosurg 2006; 108: 178-186.

8. Ellison DW, Hawkins C, Jones DTW, Onar-Thomas A, Pfister SM, Reifenberger G, Louis DN. CIMPACT-NOW update 4: diffuse gliomas characterized by MYB, MYBL1, or FGFR1 alterations or BRAF(V600E) mutation. Acta Neuropathol 2019; 137: 683-687.

9. Erguvan-Onal R, Ates O, Onal C, Aydin NE, Kocak A. Gliofibroma: an incompletely characterized tumor. Tumori 2004; 90 : 157-160.

10. EscalanteAbrilPA,SalazarMF,LopezGarciaNL,MadrazoMoyaMN, Zamora Guerra YU, Mata Mendoza YG, Gomez Apo E, Chavez Macias LG. WHO grade IV gliofibroma: a grading label denoting malignancy for an otherwise commonly misinterpreted neoplasm. J Pathol Transl Med 2015; 49: 325-330.

11. Friede RL. Gliofibroma. A peculiar neoplasia of collagen forming glia-like cells. J Neuropathol Exp Neurol 1978; 37: 300-313.

12. Gargano P, Zuccaro G, Lubieniecki F. Intracranial gliofibroma: a case report and review of the literature. Case Rep Pathol 2014; 2014: 165025.

13. Gessi M, Zur Muhlen A, Hammes J, Waha A, Denkhaus D, Pietsch T. Genome-wide DNA copy number analysis of desmoplastic infantile astrocytomas and desmoplastic infantile gangliogliomas. J Neuropathol Exp Neurol 2013; 72: 807-815. 
14. Goyal S, Puri T, Gunabushanam G, Sharma MC, Sarkar C, Julka PK, Rath GK. Gliofibroma: a report of three cases and review of literature. Acta Oncol 2007; 46: 1202-1204.

15. Iglesias JR, Richardson EP, Jr., Collia F, Santos A, Garcia MC, Redondo C. Prenatal intramedullary gliofibroma. A light and electron microscope study. Acta Neuropathol 1984; 62: 230234.

16. Iglesias-Rozas JR, Kraus-Huonder B, Michilli R, Tzonos S, Bader H, Chmelar C. Intracerebral gliofibroma. Dtsch Med Wochenschr 1992; 117: 1918-1922.

17. Jones MC, Diaz V, D’Agustini M, Altamirano E, Baglieri N, Drut R. Gliofibroma: report of four cases and review of the literature. Fetal Pediatr Pathol 2016; 35: 50-61.

18. Kaneva K, Yeo KK, Hawes D, Ji J, Biegel JA, Nelson MD, Bluml S, Krieger MD, Erdreich-Epstein A. Rare pediatric invasive gliofibroma has BRAFV600E mutation and transiently responds to targeted therapy before progressive clonal evolution. JCO Precis Oncol 2019; 3: PO.18.00138.

19. Kang H, Kim JW, Se YB, Park SH. Adult intracranial gliofibroma: a case report and review of the literature. J Korean Neurosurg Soc 2016; 59: 302-305.

20. Kim NR, Suh YL, Shin HJ, Park IS. Gliofibroma with extensive calcified deposits. Clin Neuropathol 2003; 22: 14-22.

21. Kros JM, Delwel EJ, De Jong TH, Tanghe HL, Van Run PR, Vissers K, Alers JC. Desmoplastic infantile astrocytoma and ganglioglioma: a search for genomic characteristics. Acta Neuropatho 2002; 104: 144-148.

22. Louis DN, Perry A, Reifenberger G, Von Deimling A, Figarella-Branger D, Cavenee WK, Ohgaki H, Wiestler OD, Kleihues P, Ellison DW. The 2016 World Health Organization classification of tumors of the central nervous system: a summary. Acta Neuropathol 2016; 131: 803-820.

23. Louis DN, Von Deimling A, Dickersin GR, Dooling EC, Seizinger BR. Desmoplastic cerebral astrocytomas of infancy: a histopathologic, immunohistochemical, ultrastructural, and molecular genetic study. Hum Pathol 1992; 23: 1402-1409.

24. Matsumura A, Takano S, Nagata M, Anno I, Nose T. Cervical intramedullary gliofibroma in a child: a case report and review of the literature. Pediatr Neurosurg 2002; 36: 105-110.

25. Merk BC, Owens JL, Lopes MB, Silva CM, Hussaini IM. STAT6 expression in glioblastoma promotes invasive growth. BMC Cancer 2011; 11: 184

26. Molenkamp G, Riemann B, Kuwert T, Strater R, Kurlemann G, Schober O, Jurgens $\mathrm{H}$, Wolff JE. Monitoring tumor activity in low grade glioma of childhood. Klin Padiatr 1998; 210: 239-242.

27. Nomura M, Hasegawa M, Kita D, Yamashita J, Minato H, Nakazato Y. Cerebellar gliofibroma with numerous psammoma bodies. Clin Neurol Neurosurg 2006; 108: 421-425.

28. Prayson RA. Gliofibroma: a distinct entity or a subtype of des moplastic astrocytoma? Hum Pathol 1996; 27: 610-613.

29. Prayson RA. Disseminated spinal cord astrocytoma with features of gliofibroma: a review of the literature. Clin Neuropathol 2013; 32: 298-302.

30. Reinhardt V, Nahser HC. Gliofibroma originating from temporoparietal hamartoma-like lesions. Clin Neuropathol 1984; 3 131-138.
31. Rushing EJ, Thompson LD, Mena H. Malignant transformation of a dysembryoplastic neuroepithelial tumor after radiation and chemotherapy. Ann Diagn Pathol 2003; 7: 240-244.

32. Ryall S, Tabori U, Hawkins C. Pediatric low-grade glioma in the era of molecular diagnostics. Acta Neuropathol Commun 2020; 8: 30 .

33. Sarkar R, Yong WH, Lazareff JA. A case report of intraventricular gliofibroma. Pediatr Neurosurg 2009; 45: 210-213.

34. Schober R, Bayindir C, Canbolat A, Urich H, Wechsler W. Gliofibroma: immunohistochemical analysis. Acta Neuropathol 1992; 83: 207-210.

35. Sharma MC, Gaikwad S, Mehta VS, Dhar J, Sarkar C. Gliofibroma: mixed glial and mesenchymal tumour. Report of three cases. Clin Neurol Neurosurg 1998; 100: 153-159.

36. Snipes GJ, Steinberg GK, Lane B, Horoupian DS. Gliofibroma. Case report. J Neurosurg 1991; 75: 642-646.

37. Suarez CR, Raj AB, Bertolone SJ, Coventry S. Carboplatinum and vincristine chemotherapy for central nervous system gliofibroma: case report and review of the literature. J Pediatr Hematol Oncol 2004; 26: 756-760

38. Vazquez M, Miller DC, Epstein F, Allen JC, Budzilovich GN. Glioneurofibroma: renaming the pediatric "gliofibroma": a neoplasm composed of Schwann cells and astrocytes. Mod Pathol 1991; 4: 519-523.

39. Windisch TR, Naul LG, Bauserman SC. Intramedullary gliofibroma: MR, ultrasound, and pathologic correlation. J Comput Assist Tomogr 1995; 19: 646-648.

40. Yoshida K, Sato K, Kubota T, Takeuchi H, Kitai R, Kashiwara K. Supratentorial desmoplastic ependymoma with giant ependymal rosettes. Clin Neuropathol 2000; 19: 186-191.

41. Zhang J, Wu G, Miller CP, Tatevossian RG, Dalton JD, Tang B, Orisme W, Punchihewa C, Parker M, Qaddoumi I, Boop FA, Lu C, Kandoth C, Ding L, Lee R, Huether R, Chen X, Hedlund E, Nagahawatte P, Rusch M, Boggs K, Cheng J, Becksfort J, Ma J, Song G, Li Y, Wei L, Wang J, Shurtleff S, Easton J, Zhao D, Fulton RS, Fulton LL, Dooling DJ, Vadodaria B, Mulder HL, Tang C, Ochoa K, Mullighan CG, Gajjar A, Kriwacki R, Sheer D, Gilbertson RJ, Mardis ER, Wilson RK, Downing JR, Baker SJ, Ellison DW, St. Jude Children's Research Hospital-Washington University Pediatric Cancer Genome Project. Whole-genome sequencing identifies genetic alterations in pediatric low-grade gliomas. Nat Genet 2013; 45: 602-612. 